\title{
An Attempt to Optimize ZnO-Like TCO Nanolayered Compound Thickness in Terms of a BPES-Related Physical Parameters Pondering
}

\author{
A. Boukhachem, ${ }^{1}$ A. Amlouk, ${ }^{1}$ K. Boubaker, ${ }^{2}$ M. Bouhafs, ${ }^{3}$ and M. Amlouk ${ }^{1}$ \\ ${ }^{1}$ Unité de Physique des Dispositifs à Semi-Conducteurs, Faculté des sciences de Tunis, Université de Tunis El Manar, \\ 2092 Tunis, Tunisia \\ ${ }^{2}$ Unité de Recherche MA2I, Ecole Nationale d'Ingénieurs, ENIT, BP 37, Belvédère, 1002 Tunis, Tunisia \\ ${ }^{3}$ École Supérieure de Sciences et Techniques de Tunis (E.S.S.T.T), 63 Rue Sidi Jabeur, 5100 Mahdia, Tunisia
}

Correspondence should be addressed to K. Boubaker, mmbb11112000@yahoo.fr

Received 19 July 2012; Accepted 3 August 2012

Academic Editors: S.-H. Kim and W. W. Shu

Copyright ( 2012 A. Boukhachem et al. This is an open access article distributed under the Creative Commons Attribution License, which permits unrestricted use, distribution, and reproduction in any medium, provided the original work is properly cited.

Recently, metal oxide nanolayered semiconductors revealed their increasing usefulness as UV detectors and transparent conductors in optoelectronic devices. In the present paper, a simple fabrication process has been carried out to prepare layered TCO compounds with different controlled thicknesses. Conjoint physical investigations allowed discussion of the validity of optimality in terms of geometrical parameters. A synthetic function based on pondered physical parameters was a practical guide to reach optimality.

\section{Introduction}

Recently, several nanolayered transparent conducting oxides TCO have been investigated [1-20] for their interesting optical, mechanical, and electrical performance and besides all their high optical transparence in the visible domain. Commonly, these oxides could be synthesized using several methods [21,22] like reactive evaporation, electron beam evaporation (EBE), pulsed laser deposition (PLD), chemical vapor deposition (CVD), sol-gel coating, and chemical spray pyrolysis.

In this study, TCO have been prepared by spray pyrolysis technique using, as main precursor, a solution of zinc acetate dissolved in deionized water. Several characterization techniques such as XRD, optical spectra, and hardness investigations have been applied to differently thick obtained samples [23-26]. The thickness-dependent performance of the different as-grown layers has been additionally investigated in terms of mechanical, optothermal and microhardness behaviours. The existence of an optimal thickness was investigated using the BPES protocol and compared to some recently published results [27].

\section{Experiment}

2.1. $\mathrm{ZnO}$ Layers Elaboration. $\mathrm{ZnO}$ thin layers have been prepared by the technique of chemical reactive technique in liquid phase spray. The obtained layers' structural and morphological properties, as well as synthesising details, have been recently published [23-26].

$\mathrm{ZnO}$ thin films having different thicknesses were obtained on $20 \times 10 \times 3 \mathrm{~mm}^{3}$ Pyrex glass substrates by spraying an aqueous solution (mixture of water and Propanol 2) containing zinc acetate $\left(\mathrm{Zn}\left(\mathrm{CH}_{3} \mathrm{CO}_{2}\right)_{2}\right)$ and a small amount of acetic acid, in order to avoid the precipitation of zinc hydroxide $\mathrm{Zn}(\mathrm{OH})_{2}$. The solution and gas (Nitrogen) flow rates were kept constant at $2 \mathrm{~cm}^{3} \cdot \mathrm{min}^{-1}$ and $4.01 \mathrm{~min}^{-1}$, respectively, corresponding to a minispray pyrolysis. The substrate temperature $T \mathrm{~s}$, of the order of $460^{\circ} \mathrm{C}$ (optimal value under the given experimental conditions, as already confirmed by A. Amlouk et al. $[23,24])$, was used to prepare these films according to in-room precedent successful attempts of deposition by the spray pyrolysis process [2834]. Six samples have been elaborated: with thicknesses $d=$ $0.16,0.4,0.58,0.64,0.84$, and $1.03 \mu \mathrm{m}$, respectively. 

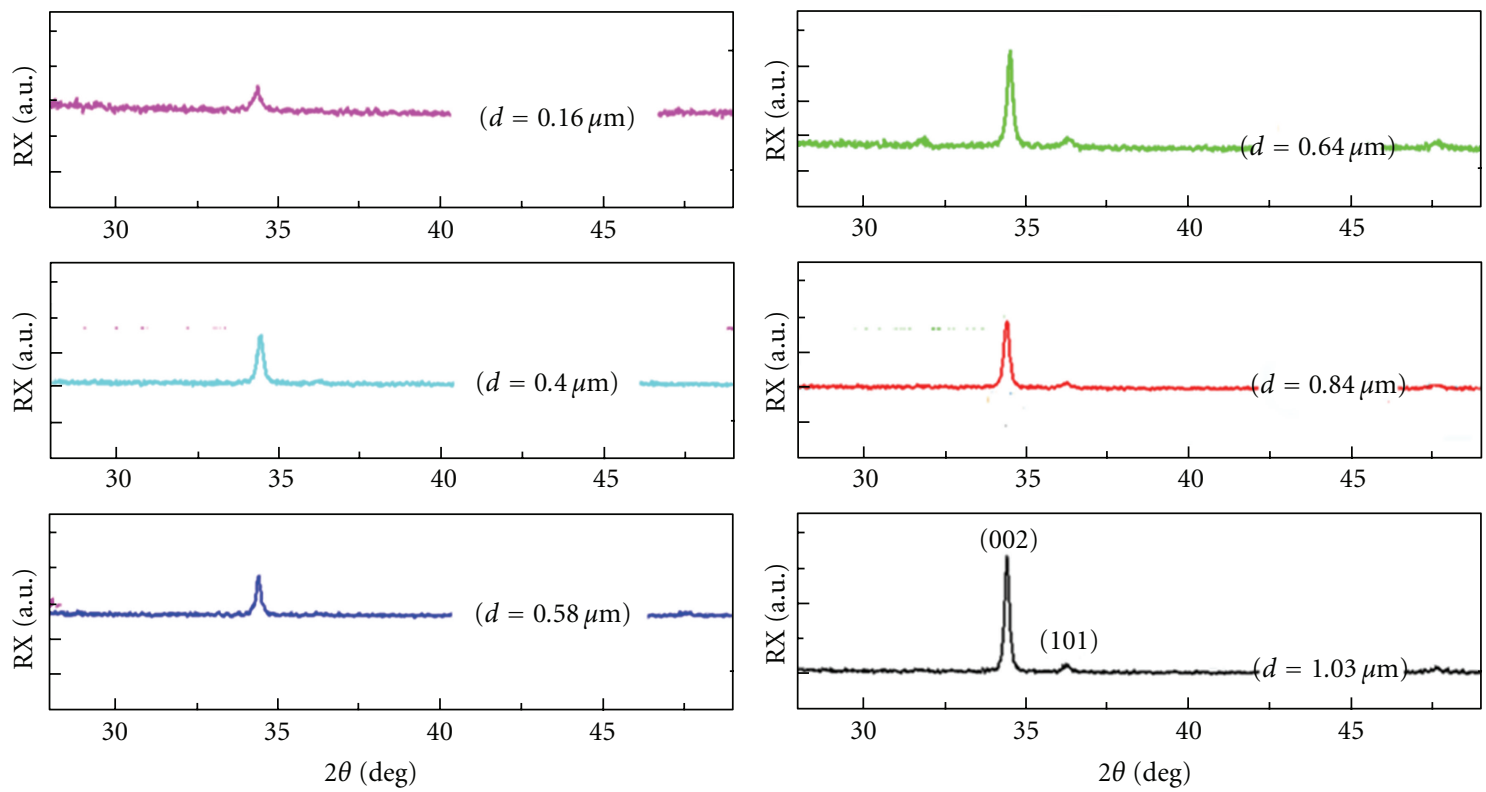

FIGURE 1: XRD Diagrams (ZnO layers $d=0.16,0.4,0.58,0.64,0.84$, and $1.03 \mu \mathrm{m}$ ).

2.2. Characterization Techniques. Precedent X-ray diffraction analyses of the prepared layers were performed by a coppersource diffractometer (Analytical $X$ Pert PROMPD), with the wavelength $(\lambda=1.54056 \AA)$. The optical transmission and reflection measures have been achieved using a common spectrophotometer (Shimadzu UV 3100S) equipped with an integrating sphere (LISR 3200). The spectrophotometer consists of double-beam monochromator (UV-2OOS, Shimazdu apparatus) with monochromatization performed by two flat silicon crystals in Laue diffraction on the (111) plane and enough energy to make several types of accurate measures in a wide wave-length range $(220-1800 \mathrm{~nm})$. The loadindependent hardness measurements have been performed by a standard Microhardness Vickers (Hv) apparatus.

\section{Already Achieved Analyses and Discussion}

XRD diagram analysis (Figure 1) shows that the thinner layers $(d<0.6 \mu \mathrm{m})$ develop an exclusive preferred orientation of the crystallites with respect to the (002) reflection. Differently, the thicker ones, are identified, beyond the (002) peak, by an extra XRD peak: (101).

These features correspond to the hexagonal würtzite system (JCPDS card, file $\mathrm{n}^{\circ}: 361451\langle a=3.24982, c=$ $5.20661 \AA\rangle$ ), which is generally associated, as stated by Dubey et al. [35], Kumar et al. [34] and Paraguay et al. [36], with the appearance of irregularly spaced pores. This structure results in an increasing level of surface roughness $[37,38]$. It seems that a minimal thickness is needed for the stability of a regular hexagonal würtzite system. Beyond this minimal thicknesses, the epitaxial growth seems to occur with no alteration in the lattice parameters, as discussed earlier by Kaeble [38].

The already published reflection $R(\lambda)$ spectra [27] presented an accurate guide for estimating the thickness of each sample. In fact, the oscillating behavior of the $\lambda$-dependent reflection in the visible domain traduces the fact that the reflectance reaches discrete $p$-indexed maximal values $R_{\max }^{(p)}$ for some particular $p$-indexed values $\lambda_{\max }^{p}$ of the wavelength [39-42].

These $p$ occurrences are traduced by the following equations:

$$
\begin{gathered}
\sqrt{R_{\max }^{(p)}(\lambda)}=\frac{n\left(\lambda_{\max }^{p}\right)^{2}-n_{S}}{n\left(\lambda_{\max }^{p}\right)^{2}+n_{S}}, \\
n\left(\lambda_{\max }^{p}\right)=\sqrt{n_{S} \frac{1+\sqrt{R_{\max }(\lambda)}}{1-\sqrt{R_{\max }(\lambda)}}},
\end{gathered}
$$

where $n_{s}$ is the substrate optical index $\left(n_{s} \approx 1.55\right)$ and $n$ is the layer $\lambda$-dependent optical index deduced from the optical transmission-reflection spectra.

The knowledge of two successive couples of maximal values $[40,41]$ allows calculating the film's thickness $d$ :

$$
d=\frac{\lambda_{\max }^{p} \lambda_{\max }^{p+1}}{2\left(\lambda_{\max }^{p} n\left(\lambda_{\max }^{p+1}\right)-\lambda_{\max }^{p+1} n\left(\lambda_{\max }^{p}\right)\right)} .
$$

\section{Additional Mechanical Moh's Hardness and Optothermal Investigations}

Hardness is the resistance offered by a material to localized plastic deformation caused by scratching or indentation. Load-independent Moh's hardness $\left(H_{M}\right)$ estimated from the scratch test is on the Moh's scale, which could be calculated out from the Microhardness Vickers $\left(H_{v}\right)$ using the formula [42]:

$$
H_{M}=0.675 \times \sqrt[3]{H_{V}} .
$$




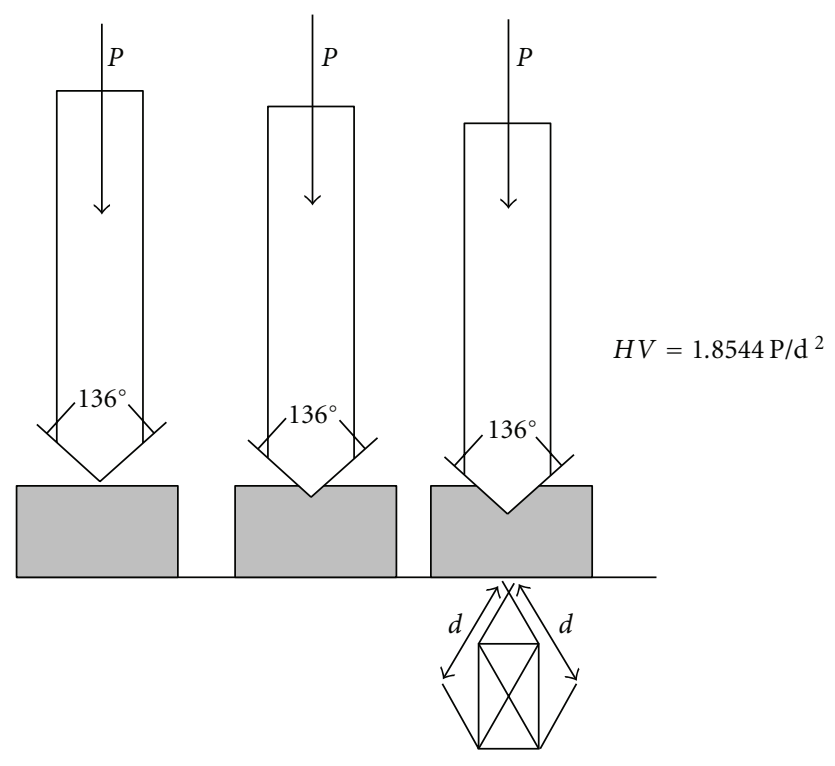

Figure 2: Vickers test disposal.

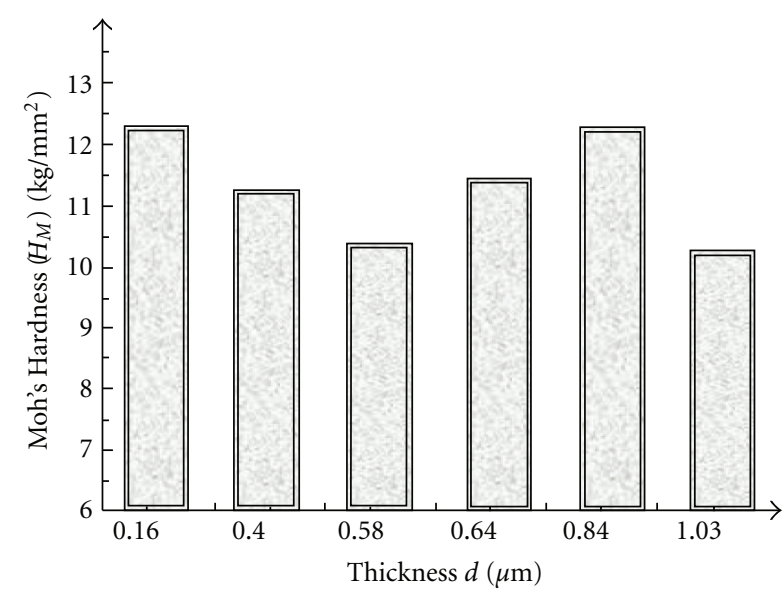

Figure 3: The thickness-dependent Moh's Hardness.

The set of hardness of measurements has been performed using a common diamond-pyramidal-indenter under a prefixed load. The obtained imprints dimensions have been exploited for yielding the Microhardness Vickers $\left(H_{v}\right)$ of each sample as a synthetic value at room temperature using a Vickers diamond pyramidal indenter equipped with squared pyramid with $136^{\circ}$ as summit angle (Figure 2). During experiment, it has been verified experimentally that the indenter penetration depth was always less than the layer's thickness. The experimental values are gathered in (Figure 3).

The differences noticed in (Figure 3) can be explained, for the thinner layers, by the hardness contribution of the Pyrex substrate. The remaining layers, develop a mean hardness varying in a narrow interval. If the contribution of the substrate can be omitted, it seems that there is a maximum hardness zone situated in the interval of thickness $[0.60-0.80 \mu \mathrm{m}]$. This noticed feature is probably due to the establishment of the hexagonal würtzite system which is harder to demolish than unorganized structures $[33,34,36$, 37].

The effective absorptivity $\hat{\alpha}$, as defined in precedent studies $[35,43]$, is the mean normalized absorbance weighted by $I(\tilde{\lambda})_{\text {AM1.5 }}$, the solar standard irradiance:

$$
\begin{gathered}
\hat{\alpha}=\frac{\int_{0}^{1} I(\tilde{\lambda})_{\mathrm{AM} 1.5} \times \alpha(\tilde{\lambda}) d \tilde{\lambda}}{\int_{0}^{1} I(\tilde{\lambda})_{)_{\mathrm{AM} 1.5}} d \tilde{\lambda}}, \\
\lambda \in\left[\lambda_{\min }, \lambda_{\max }\right] \Longleftrightarrow \tilde{\lambda} \in[0,1], \\
\lambda_{\min }=300.0 \mathrm{~nm} ; \quad \lambda_{\max }=1800.0 \mathrm{~nm},
\end{gathered}
$$

where $I(\tilde{\lambda})_{\mathrm{AM} 1.5}$ is the reference solar spectral irradiance, fitted using the Boubaker polynomials expansion scheme BPES [44-47]: $I(\tilde{\lambda})=\left[\left(1 / 2 N_{0}\right) \sum_{n=1}^{N_{0}} \theta_{n} \cdot B_{4 n}\left(\tilde{\lambda} \times \beta_{n}\right)\right]$, where $\beta_{n}$ are the Boubaker polynomials $[45,46] B_{4 n}$ minimal positive roots, $\theta_{n}$ are given coefficients, $N_{0}$ is a given integer, $\alpha(\tilde{\lambda})$ is the normalized absorbance spectrum, and $\tilde{\lambda}$ is the normalised wavelength.

The normalized absorbance spectrum $\alpha(\tilde{\lambda})$ is deduced from the BPES by establishing a set of $N$ experimental measured values of the transmittance-reflectance vector $\left.\left(T_{i}\left(\widetilde{\lambda}_{i}\right) ; R_{i}\left(\widetilde{\lambda}_{i}\right)\right)\right|_{i=1 \ldots N}$ versus the normalized wavelength $\left.\tilde{\lambda}_{i}\right|_{i=1 \ldots N}$. Then the system (5) is set:

$$
\begin{aligned}
& R(\tilde{\lambda})=\left[\frac{1}{2 N_{0}} \sum_{n=1}^{N_{0}} \xi_{n} \times B_{4 n}\left(\tilde{\lambda} \times \beta_{n}\right)\right], \\
& T(\tilde{\lambda})=\left[\frac{1}{2 N_{0}} \sum_{n=1}^{N_{0}} \xi_{n}^{\prime} \times B_{4 n}\left(\tilde{\lambda} \times \beta_{n}\right)\right],
\end{aligned}
$$

where $\beta_{n}$ are the $4 n$-Boubaker polynomials $B_{4 n}$ minimal positive roots, $N_{0}$ is a given integer and $\xi_{n}$ and $\xi_{n}^{\prime}$ are coefficients determined through the Boubaker polynomials expansion scheme (BPES).

The normalized absorbance spectrum $\alpha(\tilde{\lambda})$ is deuced from the relation:

$$
\alpha(\tilde{\lambda})=\frac{1}{d \sqrt[4]{2}} \cdot \sqrt[4]{\left(\ln \frac{1-R(\tilde{\lambda})}{T(\tilde{\lambda})}\right)^{4}+\left(2 \ln \frac{1-R(\tilde{\lambda})}{\sqrt{T(\tilde{\lambda})}}\right)^{4}},
$$

where $d$ is the layer thickness.

The obtained value of normalized absorbance spectrum $\alpha(\tilde{\lambda})$ is a final guide to the determination of the effective absorptivity $\hat{\alpha}$ through (4).

The Amlouk-Boubaker optothermal expansivity $\Psi_{\mathrm{AB}}$ is a thermophysical parameter defined in precedent studies [35, 43-49], as a 3D expansion velocity of the transmitted heat inside the material. It is expressed in $\mathrm{m}^{3} \mathrm{~s}^{-1}$, and calculated by the following:

$$
\Psi_{\mathrm{AB}}=\frac{D}{\hat{\alpha}},
$$


where $D$ is the thermal diffusivity and $\hat{\alpha}$ is the effective absorptivity.

This parameter gathers two totally different parameters: the thermal diffusivity which is a material intrinsic property, and the effective absorptivity which links the material response to the solar spectrum. The Amlouk-Boubaker optothermal expansivity $\Psi_{\mathrm{AB}}$ presents the advantage of describing the conjoint thermal-optical response of the material to the whole spectrum, not only to monochromatic excitation.

As optimality is a complex notion when several physical parameters are involved, a synthetic aggregate is proposed on the bases of both Amlouk-Boubaker optothermal expansivity $\Psi_{\mathrm{AB}}$ and load-independent Moh's Hardness $H_{M}$. For this purpose, and according to the definition of the Boubaker polynomials expansion scheme (BPES) [44-47], the conjoint values of the $N(N=6)$ obtained $d$ dependent optothermal expansivity $\Psi_{\mathrm{AB}}$ and Moh's Hardness $H_{M}$ are ordered as $\left.\left(\left(\Psi_{\mathrm{AB}}\right)_{k},\left(H_{M}\right)_{k}\right)\right|_{k=1 \ldots N}$ within the ranges $\left[\left(\Psi_{\mathrm{AB}}\right)_{\min },\left(\Psi_{\mathrm{AB}}\right)_{\max }\right]$ and $\left[\left(H_{M}\right)_{\min },\left(H_{M}\right)_{\max }\right]$. Consecutively, the two normalized variables $\widetilde{\Psi}_{A B}, \widetilde{d}$ and $\widetilde{H}_{M}$ are defined as follows:

$$
\begin{gathered}
\tilde{\Psi}_{A B}=\frac{\Psi_{\mathrm{AB}}-\left(\Psi_{\mathrm{AB}}\right)_{\min }}{\left(\Psi_{\mathrm{AB}}\right)_{\max }-\left(\Psi_{\mathrm{AB}}\right)_{\min }}, \\
\tilde{H}_{M}=\frac{H_{M}-\left(H_{M}\right)_{\min }}{\left(H_{M}\right)_{\max }-\left(H_{M}\right)_{\min }}, \\
\tilde{d}=\frac{d-d_{\min }}{d_{\max }-d_{\min }} .
\end{gathered}
$$

The thickness is hence expressed as follows:

$$
\begin{aligned}
\tilde{d}\left(\Psi_{\mathrm{AB}}, H_{M}\right)= & \mid \frac{1}{2 N_{0}} \sum_{q=1}^{N_{0}} \xi_{q} \cdot B_{4 q}\left(\widetilde{\Psi}_{A B} \alpha_{q}\right) \\
& -\frac{1}{2 N_{0}} \sum_{q=1}^{N_{0}} \xi_{q}^{\prime} \cdot B_{4 q}\left(H_{M} \alpha_{q}\right) \mid,
\end{aligned}
$$

where $\alpha_{q}$ is $4 \mathrm{q}$-Boubaker polynomial minimal root [45], $N_{0}$ is a prefixed integer, $\left.\xi_{q}\right|_{q=1 \ldots N_{0}}$ and $\left.\xi_{q}^{\prime}\right|_{q=1 \ldots N_{0}}$ are unknown coefficients. The final step consists of computing (using Matlab subroutine SSO45) the set of coefficients $\left.\left(\xi_{q, \mathrm{opt}}, \xi_{q, \mathrm{opt}}^{\prime}\right)\right|_{q=1 \ldots N_{0}}$ which minimizes the real functional $\Psi(x)$ :

$$
\begin{aligned}
\Psi\left(N, N_{0}\right)=\sum_{k=1}^{N} \mid & \frac{1}{2 N_{0}} \sum_{q=1}^{N_{0}} \xi_{q} \cdot B_{4 q}\left(\widetilde{\Psi}_{A B} \alpha_{q}\right) \\
& -\frac{1}{2 N_{0}} \sum_{q=1}^{N_{0}} \xi_{q}^{\prime} \cdot B_{4 q}\left(H_{M} \alpha_{q}\right) \mid .
\end{aligned}
$$

The obtained solution $d\left(\Psi_{\mathrm{AB}}, H_{M}\right)$, for $N=6, N_{0}=20$, is normalized then plotted in Figure 4.

\section{Discussion and Perspectives}

A study has been recently presented by Lee et al. [48] on the thickness-related optimization inside $\mathrm{IZO} / \mathrm{ZnO} / \mathrm{PET}$

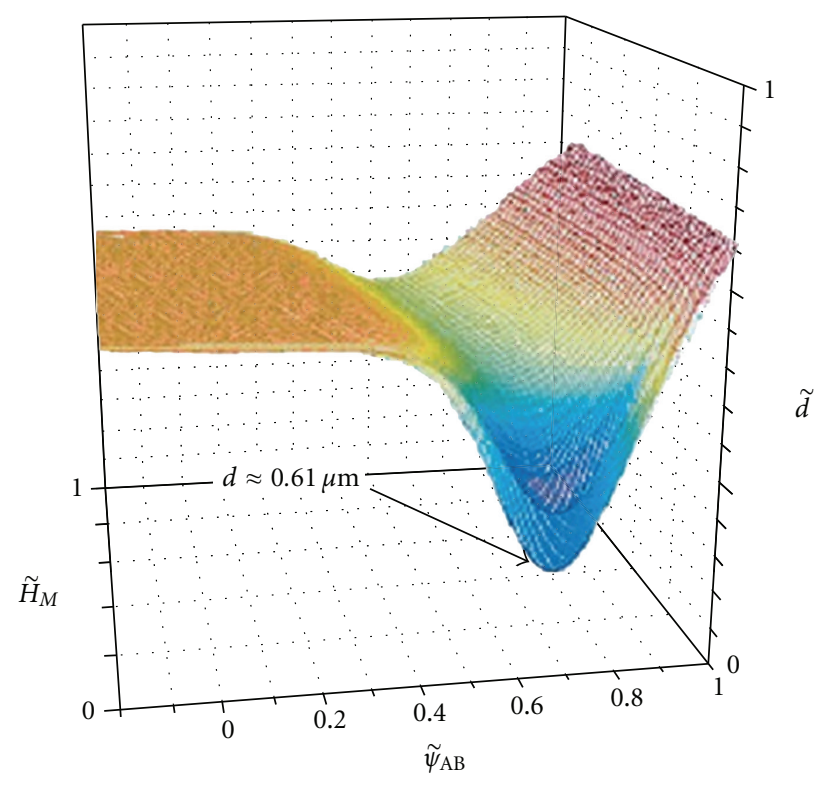

Figure 4: Global optimality curve.

multilayered structures. The authors have prospected the TCO-induced exclusive electrical performance (carriers' concentration, mobility...) and conjectured the existence of an eventual optimal thickness. Earlier, Shan and Yu [49] attempted to verify optimality for a thickness around $500 \mathrm{~nm}$. These intriguing observations have been confirmed through the features of Figure 4. In fact, the optimality is obtained for the lowest point $(d \approx 0.61 \mu \mathrm{m})$. This optimality is deduced by estimating the thickness which corresponds to a maximum of the load-independent Moh's Hardness conjointly with a minimal value of AmloukBoubaker optothermal expansivity $\Psi_{\mathrm{AB}}$. For this purpose, an optimisation protocol has been established for calculating a pondered function $\widetilde{F}$ of Moh's Hardness and AmloukBoubaker optothermal expansivity $\Psi_{\mathrm{AB}}$ with positive weights for Moh's Hardness and negative ones for $\Psi_{\mathrm{AB}}$. For each value of the measured couple (Moh's Sardness, $\Psi_{\mathrm{AB}}$ ), the correspondent values of the thickness $d$ along with the test function $\widetilde{F}$ were recorded. Optimality was reached for the value of which corresponded to maximum value of $\tilde{F}$.

Moreover, the mechanical study revealed that, in order to reach the intrinsic hardness value of the layer (required for TCO applications in shock circumstances or aggressive mediums...), while avoiding substrate effects, one must either use a load as low as possible, or ensure a thickness exceeding the micrometer. Still, these values can be refined by using the nanohardness measurements to obtain the accurate hardness values of such thin films.

\section{Conclusion}

In summary, we have discussed the effects of the controlled thickness of TCO thin films prepared using a simplified and 
already optimized spray pyrolysis setup. Besides commonly considered parameters of the obtained layers, new and original characterization protocols have been carried out. It has been stated that an optimal $\mathrm{ZnO}$ thin film thickness is required for TCO purposes via simultaneous morphologicalstructural-physical investigations.

\section{References}

[1] M. Regragui, V. Jousseaume, M. Addou, A. Outzourhit, J. C. Bernéde, and B. El Idrissi, "Electrical and optical properties of $\mathrm{WO}_{3}$ thin films," Thin Solid Films, vol. 397, no. 1-2, pp. 238 243, 2001

[2] F. Yubero, V. M. Jiménez, and A. R. González-Elipe, “Optical properties and electronic transitions of $\mathrm{SnO}_{2}$ thin films by reflection electron energy loss spectroscopy," Surface Science, vol. 400, no. 1-3, pp. 116-126, 1998.

[3] E. G-Berasategui, S. J. Bull, and T. F. Page, "Mechanical modelling of multilayer optical coatings," Thin Solid Films, vol. 447-448, pp. 26-32, 2004.

[4] R. Thokala and J. Chaudhuri, "Calculated elastic constants of wide band gap semiconductor thin films with a hexagonal crystal structure for stress problems," Thin Solid Films, vol. 266, no. 2, pp. 189-191, 1995.

[5] R. B. Patil, R. K. Puri, and V. Puri, "Improvement in the mechanical properties of tin oxide thin films due to vapour chopping," Journal of Alloys and Compounds, vol. 462, no. 1-2, pp. 235-239, 2008.

[6] P. M. Gorley, V. V. Khomyak, S. V. Bilichuk, I. G. Orletsky, P. P. Horley, and V. O. Grechko, " $\mathrm{SnO}_{2}$ films: formation, electrical and optical properties," Materials Science and Engineering B, vol. 118, no. 1-3, pp. 160-163, 2005.

[7] D. Davazoglou and A. Donnadieu, "Structure and optical properties of $\mathrm{WO}_{3}$ thin films prepared by chemical vapour deposition," Thin Solid Films, vol. 147, no. 2, pp. 131-142, 1987.

[8] S. W. Xue, X. T. Zu, W. L. Zhou et al., "Effects of post-thermal annealing on the optical constants of $\mathrm{ZnO}$ thin film," Journal of Alloys and Compounds, vol. 448, no. 1-2, pp. 21-26, 2008.

[9] T. Serin, N. Serin, S. Karadeniz, H. Sari, N. Tuğluoğlu, and O. Pakma, "Electrical, structural and optical properties of $\mathrm{SnO}_{2}$ thin films prepared by spray pyrolysis," Journal of NonCrystalline Solids, vol. 352, no. 3, pp. 209-215, 2006.

[10] D. Souri and K. Shomalian, "Band gap determination by absorption spectrum fitting method (ASF) and structural properties of different compositions of $(60-x) \mathrm{V}_{2} \mathrm{O}_{5}-$ $40 \mathrm{TeO}_{2-X} \mathrm{Sb}_{2} \mathrm{O}_{3}$ glasses," Journal of Non-Crystalline Solids, vol. 355, no. 31-33, pp. 1597-1601, 2009.

[11] J. C. Manifacier, "Thin metallic oxides as transparent conductors," Thin Solid Films, vol. 90, no. 3, pp. 297-308, 1982.

[12] A. Mosbah, A. Moustaghfir, S. Abed et al., "Comparison of the structural and optical properties of zinc oxide thin films deposited by d.c. and r.f. sputtering and spray pyrolysis," Surface and Coatings Technology, vol. 200, no. 1-4, pp. 293296, 2005.

[13] Y. Villachon-Renard, G. Leveque, A. Abdellaoui, and A. Donnadieu, "Optical constants of electrochromic polycrystalline $\mathrm{WO}_{3}$ thin films prepared by chemical vapour deposition," Thin Solid Films, vol. 203, no. 1, pp. 33-39, 1991.

[14] N. Kikuchi, E. Kusano, E. Kishio, and A. Kinbara, "Electrical and mechanical properties of $\mathrm{SnO}_{2}: \mathrm{Nb}$ films for touch screens," Vacuum, vol. 66, no. 3-4, pp. 365-371, 2002.
[15] G. X. Liu, F. K. Shan, W. J. Lee, B. C. Shin, H. S. Kim, and J. H. Kim, "Boron and nitrogen co-doped $\mathrm{ZnO}$ thin films for optoelectronic applications," Ceramics International, vol. 34, no. 4, pp. 1011-1015, 2008.

[16] E. Elangovan and K. Ramamurthi, "Optoelectronic properties of spray deposited $\mathrm{SnO}_{2}: \mathrm{F}$ thin films for window materials in solar cells," Journal of Optoelectronics and Advanced Materials, vol. 5, no. 1, pp. 45-54, 2003.

[17] L. Niinistö, J. Päiväsaari, J. Niinistö, M. Putkonen, and M. Nieminen, "Advanced electronic and optoelectronic materials by atomic layer deposition: an overview with special emphasis on recent progress in processing of high-k dielectrics and other oxide materials," Physica Status Solidi A, vol. 201, no. 7, pp. 1443-1452, 2004.

[18] K. Ramamoorthy, M. Jayachandran, K. Sankaranarayanan, P. Misra, L. M. Kukreja, and C. Sanjeeviraja, "Development of a novel high speed (electron-mobility) epi-n-ZnO thin films by L-MBE for III-V opto-electronic devices," Current Applied Physics, vol. 4, no. 6, pp. 679-684, 2004.

[19] H. C. Cheng, C. F. Chen, and C. C. Lee, "Thin-film transistors with active layers of zinc oxide $(\mathrm{ZnO})$ fabricated by lowtemperature chemical bath method," Thin Solid Films, vol. 498, no. 1-2, pp. 142-145, 2006.

[20] Y. Furubayashi, T. Hitosugi, Y. Yamamoto et al., "Novel transparent conducting oxide: anatase $\mathrm{Ti}_{1-x} \mathrm{Nb}_{x} \mathrm{O}_{2}$," Thin Solid Films, vol. 496, no. 1, pp. 157-159, 2006.

[21] J. Szuber, G. Czempik, R. Larciprete, and B. Adamowicz, "Comparative XPS and PYS studies of $\mathrm{SnO}_{2}$ thin films prepared by L-CVD technique and exposed to oxygen and hydrogen," Sensors and Actuators B, vol. 70, no. 1-3, pp. 177181,2000

[22] J. H. Chung, Y. S. Choe, and D. S. Kim, "Effect of low energy oxygen ion beam on optical and electrical characteristics of dual ion beam sputtered $\mathrm{SnO}_{2}$ thin films," Thin Solid Films, vol. 349, no. 1, pp. 126-129, 1999.

[23] A. Amlouk, K. Boubaker, and M. Amlouk, " $\mathrm{SnO}_{2}$ thin films morphological and optical properties in terms of the Boubaker Polynomials Expansion Scheme BPES-related Opto-Thermal Expansivity $\psi \mathrm{AB}$," Journal of Alloys and Compounds, vol. 490, no. 1-2, pp. 602-604, 2010.

[24] A. Amlouk, K. Boubaker, M. Amlouk, and M. Bouhafs, "Study of ytterbium doping effects on structural, mechanical and opto-thermal properties of sprayed $\mathrm{ZnO}$ thin films using the Boubaker Polynomials Expansion Scheme (BPES)," Journal of Alloys and Compounds, vol. 485, no. 1-2, pp. 887-891, 2009.

[25] S. Dabbous, T. Ben Nasrallah, J. Ouerfelli, K. Boubaker, M. Amlouk, and S. Belgacem, "Study of structural and optical properties of sprayed $\mathrm{WO}_{3}$ thin films using enhanced characterization techniques along with the Boubaker Polynomials Expansion Scheme (BPES)," Journal of Alloys and Compounds, vol. 487, no. 1-2, pp. 286-292, 2009.

[26] B. Ouni, J. Ouerfelli, A. Amlouk, K. Boubaker, and M. Amlouk, "Structural, mechanical and opto-thermal properties of non-crystalline SbxOy thin films," Journal of NonCrystalline Solids, vol. 356, no. 25-27, pp. 1294-1299, 2010.

[27] K. Boubaker, "Optimization of $\mathrm{ZnO}$ sheets dimension in terms of ductility, micro-indentation, mechanical resistance, Amlouk-Boubaker optothermal expansivity and crystallites size," Materials Science and Engineering A, vol. 528, no. 3, pp. 1455-1457, 2011.

[28] M. Amlouk, S. Belgacem, N. Kamoun, H. EL Houichet, and R. Bennaceur, "Study of physical-properties of indium-doped zno airless sprayed thin-layers," Annales de Chimie, vol. 19, pp. 469-477, 1994. 
[29] M. Amlouk, F. Touhari, S. Belgacem, N. Kamoun, D. Barjon, and R. Bennaceur, "Structural and acoustic properties of $\mathrm{ZnO}$ thin films prepared by spray," Physica Status Solidi A, vol. 163, no. 1, pp. 73-80, 1997.

[30] G. L. Mar, P. Y. Timbrell, and R. N. Lamb, Chemistry of Materials, vol. 5, pp. 7-14, 1995.

[31] F. Paraguay, W. Estrada, D. R. Acosta, E. Andrade, and M. Miki-Yoshida, "Growth, structure and optical characterization of high quality $\mathrm{ZnO}$ thin films obtained by spray pyrolysis," Thin Solid Films, vol. 350, no. 1, pp. 192-202, 1999.

[32] O. F. Z. Khan and P. O'Brien, “On the use of zinc acetate as a novel precursor for the deposition of $\mathrm{ZnO}$ by low-pressure metal-organic chemical vapour deposition," Thin Solid Films, vol. 173, no. 1, pp. 95-97, 1989.

[33] M. A. Lucio-López, M. A. Luna-Arias, A. Maldonado, M. D. L. L. Olvera, and D. R. Acosta, "Preparation of conducting and transparent indium-doped $\mathrm{ZnO}$ thin films by chemical spray," Solar Energy Materials and Solar Cells, vol. 90, no. 6, pp. 733741, 2006.

[34] P. M. R. Kumar, C. S. Kartha, and K. P. Vijayakumar, "Doping of spray-pyrolyzed $\mathrm{ZnO}$ thin films through direct diffusion of indiumml: structural optical and electrical studies," Journal of Applied Physics, vol. 98, no. 2, Article ID 023509, 2005.

[35] B. Dubey, T. G. Zhao, M. Jonsson, and H. Rahmanov, "A solution to the accelerated-predator-satiety Lotka-Volterra predator-prey problem using Boubaker polynomial expansion scheme," Journal of Theoretical Biology, vol. 264, no. 1, pp. 154-160, 2010.

[36] F. Paraguay, J. Morales, W. Estrada, E. Andrade, and M. MikiYoshida, "Influence of $\mathrm{Al}, \mathrm{In}, \mathrm{Cu}, \mathrm{Fe}$ and $\mathrm{Sn}$ dopants in the microstructure of zinc oxide thin films obtained by spray pyrolysis," Thin Solid Films, vol. 366, no. 1-2, pp. 16-27, 2000.

[37] B. D. Cullity, Elements of X-Ray Diffraction, Addison Wesley, 1978.

[38] E. F. Kaeble, Handbook of X-Rays, McGraw-Hill, New York, NY, USA, 1967.

[39] S. Belgacem, J. M. Saurel, and J. Bougnot, "The optical properties of sprayed CdS thin films," Thin Solid Films, vol. 92, no. 3, pp. 199-209, 1982.

[40] S. Belgacem, R. Bennaceur, J. Saurel, and J. Bougnot, "Propriétés optiques des couches minces de $\mathrm{SnO}_{2}$ et CuInS2 airless spray," Revue de Physique Appliquée, vol. 25, pp. 1245-1258, 1990.

[41] D. A. Minkov, Journal of Physics D, vol. 22, pp. 199-207, 1989.

[42] D. B. Sirdeshmukh, L. Sirdeshmukh, K. G. Subhadra, K. K. Rao, and S. B. Laxman, "Systematic hardness measurements on some rare earth garnet crystals," Bulletin of Materials Science, vol. 24, no. 5, pp. 469-473, 2001.

[43] S. Lazzez, K. B. Ben Mahmoud, S. Abroug, F. Saadallah, and M. Amlouk, "A Boubaker polynomials expansion scheme (BPES)related protocol for measuring sprayed thin films thermal characteristics," Current Applied Physics, vol. 9, no. 5, pp. 1129-1133, 2009.

[44] T. G. Zhao, L. Naing, and W. X. Yue, "Some new features of the Boubaker polynomials expansion scheme BPES," Matematicheskie Zametki, vol. 87, no. 2, pp. 175-178, 2010.

[45] A. Yildirim, S. T. Mohyud-Din, and D. H. Zhang, Computers \& Mathematics with Applications, vol. 59, pp. 2473-2488, 2010.

[46] S. A. H. A. E. Tabatabaei, T. Zhao, O. B. Awojoyogbe, and F. O. Moses, "Cut-off cooling velocity profiling inside a keyhole model using the Boubaker polynomials expansion scheme," Heat and Mass Transfer, vol. 45, no. 10, pp. 1247-1251, 2009.

[47] A. Belhadj, O. F. Onyango, and N. Rozibaeva, "Boubaker polynomials expansion scheme-related heat transfer investigation inside keyhole model," Journal of Thermophysics and Heat Transfer, vol. 23, no. 3, pp. 639-640, 2009.

[48] C. Lee, A. Park, Y. Cho, M. Park, W. I. Lee, and H. W. Kim, "Influence of $\mathrm{ZnO}$ buffer layer thickness on the electrical and optical properties of indium zinc oxide thin films deposited on PET substrates," Ceramics International, vol. 34, no. 4, pp. 1093-1096, 2008.

[49] F. K. Shan and Y. S. Yu, "Band gap energy of pure and Al-doped $\mathrm{ZnO}$ thin films," Journal of the European Ceramic Society, vol. 24, no. 6, pp. 1869-1872, 2004. 

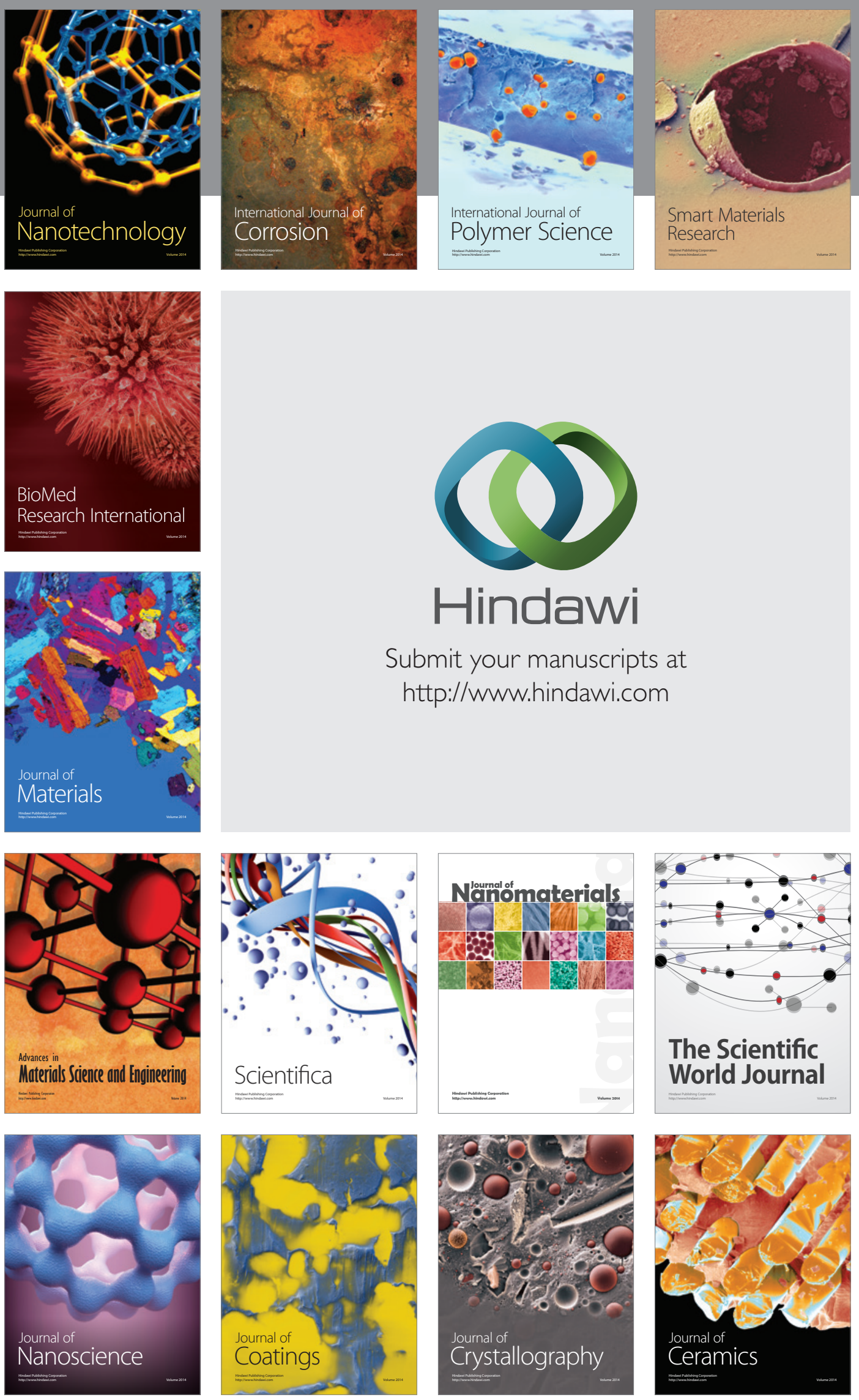

The Scientific World Journal

Submit your manuscripts at

http://www.hindawi.com

\section{World Journal}

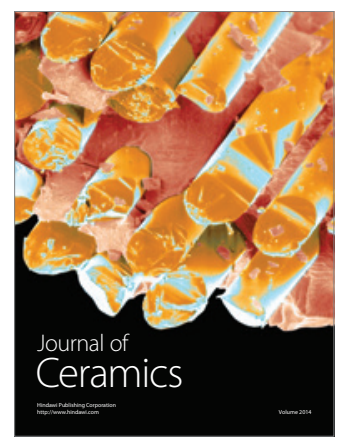

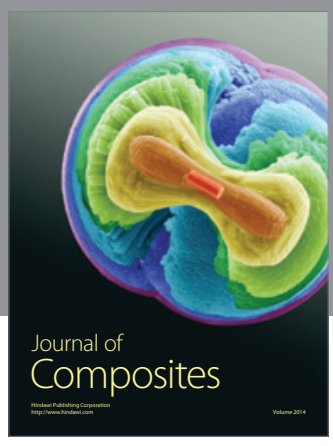
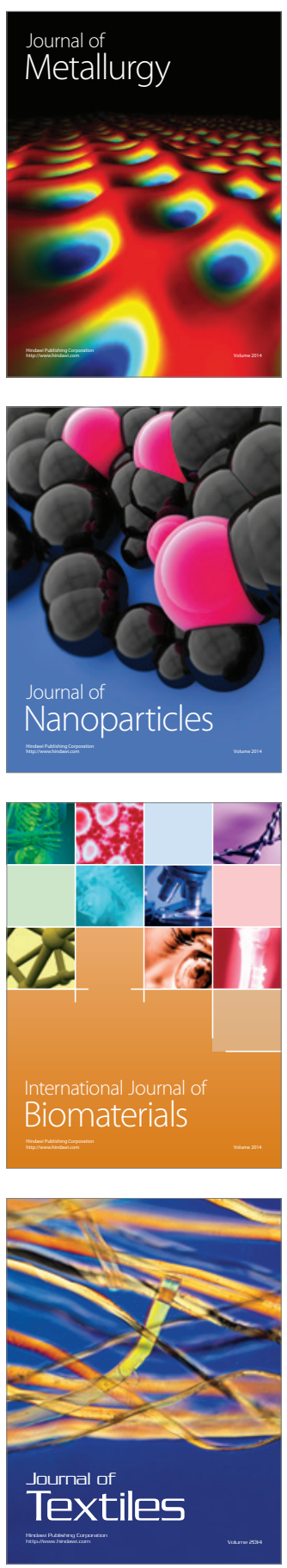